\title{
Staphylococcus aureus Pathogenicity Islands: Hijackers on the Bacteriophage Assembly Pathway.
}

Terje Dokland $^{1}$, James L. Kizziah ${ }^{1}$, Altaira D. Dearborn ${ }^{2}$, Keith A. Manning ${ }^{1}$, Erin A. Wall ${ }^{3}$, Laura Klenow $^{3}$, Laura K. Parker ${ }^{3}$, Gail E. Christie ${ }^{3}$

1. Department of Microbiology, University of Alabama at Birmingham, Birmingham, AL, U.S.A.

2. National Institute for Arthritis and Musculoskeletal and Skin Diseases, Bethesda, MD, U.S.A.

3. Department of Microbiology and Immunology, Virginia Commonwealth University, Richmond, CA, U.S.A.

Most virulence factors in Staphylococcus aureus are associated with mobile genetic elements. Bacteriophages represent the main mechanism by which such elements are mobilized and transferred, typically at low frequency (generalized transduction). S. aureus pathogenicity islands (SaPIs) engage in a kind of "molecular piracy" in which they hijack the assembly pathway of "helper" bacteriophages, such as $80 \alpha$, for encapsidation and high frequency transfer of their own genomes [1].

When a cell harboring a SaPI is infected with the appropriate helper phage, the SaPI senses the presence of the phage by interacting with an early phage gene product, leading to derepression, excision and replication of the SaPI genome. SaPIs encode several factors that interfere with the multiplication of their helpers, including a small terminase subunit (TerS) that specifically recognizes the SaPI DNA for packaging, proteins that bind to the phage terminase to prevent packaging, and factors that repress phage transcription [1, 2]. Many SaPIs, like SaPI1, also redirect the capsid assembly pathway of the helper phage to produce capsids that are smaller than the normal phage capsids and are thus unable to package complete phage genomes, commensurate with the smaller SaPI genome [1].

We previously determined the three-dimensional structures of $80 \alpha$ procapsids and mature capsids to around $9 \AA$ resolution [3]. We also showed that the SaPI1-encoded proteins CpmA and CpmB are responsible for the capsid size redirection, and that $\mathrm{CpmB}$ forms an internal scaffold in SaPI1 procapsids [4-6]. We have now determined the structures of the phage $80 \alpha$ and SaPI1 procapsids to around $3.9 \AA$ resolution and the $80 \alpha$ mature capsid to $6.5 \AA$ resolution, using high resolution instrumentation available at the Biological Science Imaging Resource (BSIR) at Florida State University (Fig. 1A-C). The new maps have allowed the capsid protein (CP) and part of the scaffolding protein (SP) and CpmB to be accurately modeled into the density, showing the specific protein-protein interactions in the shell (Fig. 1D-F). The structures show that $80 \alpha$ SP and SaPI1 CpmB share a similar "helix-and-hook" structural motif at their $\mathrm{C}$-termini that includes a conserved RIIK sequence. The two proteins bind to the same location on the inside of the capsid shell (Fig. 1D, E), suggesting that the two proteins compete directly for binding to $\mathrm{CP}$ during capsid assembly. CpmA may be required to disrupt the scaffolding core made by SP in order to allow the formation of a smaller shell. Comparison with the mature capsid shows the conformational changes that occur upon DNA packaging and capsid maturation, and suggests that scaffolding release causes a destabilization of the $\mathrm{N}$-arm helix $\alpha 1$, which rotates to initiate the expansion process (Fig. 1F) [7]. 


\section{References:}

[1] GE Christie and T Dokland, Virology 434 (2012), p. 210-221.

[2] RP Novick and G Ram, Trends Genet. 32 (2016), p. 114-126.

[3] MS Spilman et al, J. Mol. Biol. 405 (2011), p. 863-876.

[4] A Poliakov et al, J. Mol. Biol. 380 (2009), p. 465-475.

[5] AD Dearborn et al, J. Mol. Biol. 412 (2011), p. 710-722.

[6] PK Damle et al, Virology 432 (2012), p. 277-282.

[7] This project was funded by The National Institutes of Health (NIH) award R01 AI083255 to T.D. The authors acknowledge John Spear and Duncan Sousa at BSIR for assistance with cryo-EM data collection; Michael Spilman at Direct Electron, LP, for help with data processing; and Rosie Hill (UAB) for producing the virus used for the mature capsid reconstruction. BSIR was supported by NIH grants S10 OD018142 and S10 RR025080 (Kenneth Taylor, PI).
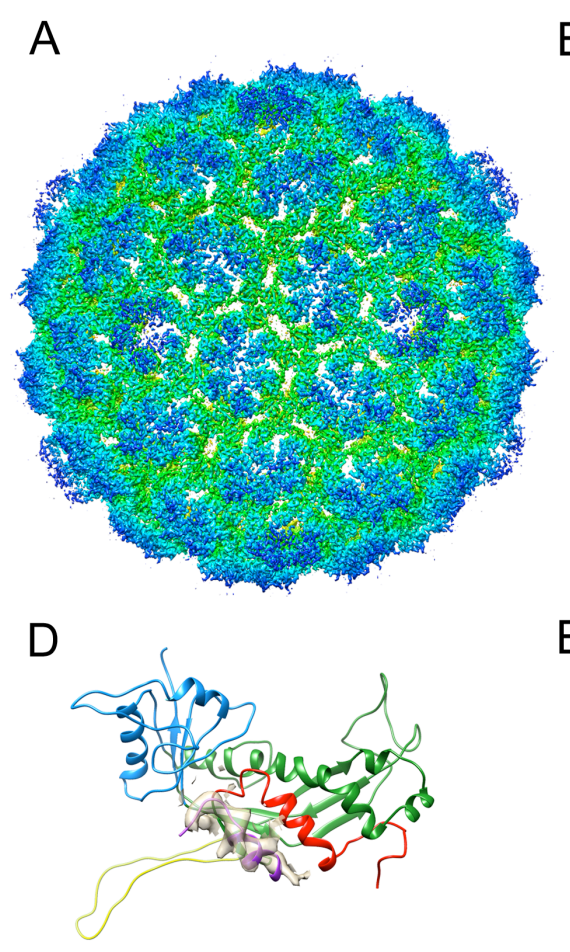

B

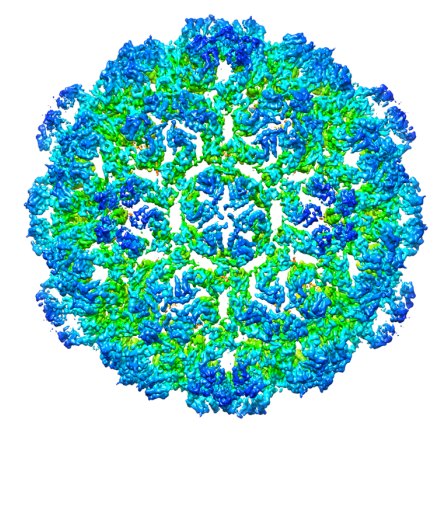

E

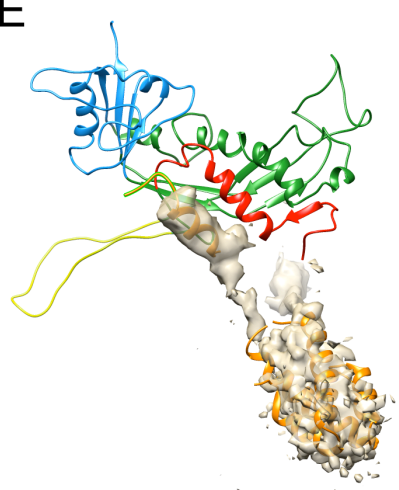

C

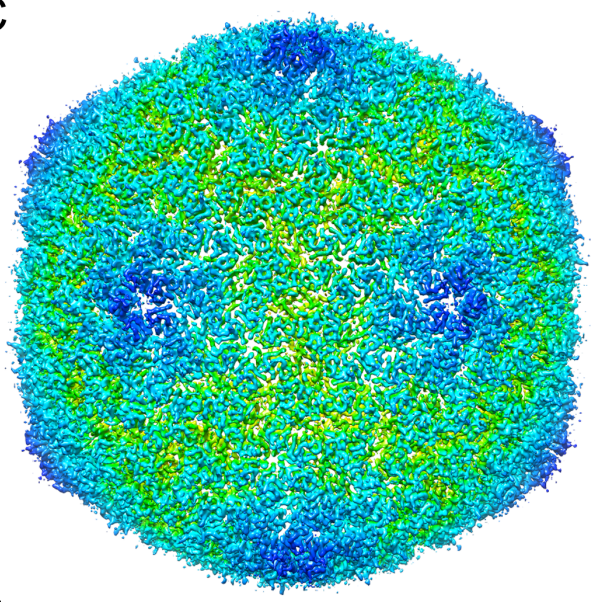

$\mathrm{F}$

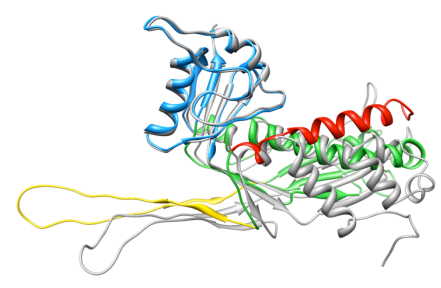

Figure 1. Surface representations of the reconstructions $(\mathrm{A}-\mathrm{C})$ and ribbon diagrams of the $\mathrm{CP}$ models (D-F) of the $80 \alpha$ procapsid (A, D), SaPI1 procapsid (B, E) and 80 $\alpha$ mature capsid (C, F). Ribbon diagram color code: red, N-arm; green, $\mathrm{P}$ domain; yellow, E loop; blue, A domain. The $80 \alpha$ model also shows part of the SP (purple) and the SaPI1 model shows CpmB (orange), with associated density superimposed. $\mathrm{CpmB}$ is partly disordered, and the dimer domain model shown is from the NMR structure. In (F), the $80 \alpha$ procapsid model is superimposed in gray. 